\title{
PCNA and Ki-67 labelling indices in pre-irradiated and post-irradiated astrocytomas: a comparative immunohistochemical analysis for evaluation of proliferative activity
}

\author{
E Pierce, R Doshi, R Deane
}

\begin{abstract}
Aim-To determine the tumour proliferative activity in a series of archival cerebral astrocytomas and compare proliferating cell nuclear antigen (PCNA) and Ki-67 labelling indices in the primary and recurring neoplasms following therapeutic radiation.

Method-Twenty eight cases of preirradiated and post-irradiated astrocytomas (ranging from WHO grades I to IV) were stained immunohistochemically using the avidin-biotin horseradish peroxidase technique. Two antibodies, PC10 and MIB-1, were used to establish the proliferating labelling indices. PC10 identifies PCNA and MIB-1 recognises the Ki-67 antigen.

Results-Both antibodies showed significantly higher labelling indices in the postirradiated specimens. However, in general, the $\mathrm{Ki}-67$ indices were lower than those for PCNA. MIB-1 immunoreactivity showed less variation and was more intense than that seen with PC10. The discrepancy between the labelling indices of the pre-irradiated and post-irradiated samples raises questions about the evolution of astrocytomas and the effects of therapeutic intervention.

Conclusions-The data may represent genetic alterations, the natural tumour course, and/or the effect of radiation. Although both of the antibodies reflected the state of growth of neoplastic cells in astrocytomas, MIB-1 was more reliable. A simple immunohistochemical method using proliferation markers does have an important role in the future care of patients with astrocytoma.

(f Clin Pathol: Mol Pathol 1998;51:90-95)
\end{abstract}

Keywords: astrocytoma; proliferation; radiation; PC10; MIB-1; PCNA; Ki-67

Neoplastic cells frequently have composite and diverse abnormalities of growth, and transformed or cancerous astrocytic cells are notorious in this respect. The pattern of growth of a tumour is an important element in determining the potential for progression and dissemination. Furthermore, planning and implementation of an effective therapeutic regimen for any tumour relies on an examination of its growth rate and state. The state of tumour cell proliferation, unlike the rate of proliferation, is a static measure that can be assessed by immunohistochemistry. ${ }^{1}$ Astrocytomas are common, devastating brain tumours. Thus, knowledge of their proliferative potential may prove vital in the prediction of recurrence or progression in histological grade and stage.

Radiotherapy following surgery, alone or with chemotherapy, is regarded as essential management in many brain tumours, including astrocytomas. Monitoring the response to radiotherapy can be difficult because the amount and action of this intricate and prognostically important agent affects and can be affected by a number of factors. There is a growing need to assess therapeutic management accurately, in order to determine and administer individual therapy. Because radiotherapy is aimed primarily at the cell cycle, the proliferative activity of the targeted cells provides a suitable area for investigation.

Proliferation markers directed at specific nuclear antigens within the cell cycle have facilitated the assessment of the growth fraction of human tumours. ${ }^{2-7}$ Two such monoclonal antibodies are $\mathrm{PC} 10^{8}$ and MIB- $1 .{ }^{9} \mathrm{PC} 10$ detects proliferating cell nuclear antigen (PCNA) and MIB-1 recognises the Ki-67 antigen within the cell cycle. Both these antigens have different levels within the different phases of the cell cycle. ${ }^{10}$ PCNA is present in all phases of the cell cycle, including $G_{0}$, and has been detected in quiescent cells, ${ }^{11}{ }^{12}$ whereas $\mathrm{Ki}-67$ is only found in the active parts of the cell cycle: $\mathrm{G}_{1}, \mathrm{G}_{2}, \mathrm{~S}$, and $M$ phases. ${ }^{13}$ The $\mathrm{Ki}-67$ antigen is rapidly catabolised after $\mathrm{M}$ phase. ${ }^{14} \mathrm{PC} 10^{15}$ and MIB- $1^{16}$ binding have been reported as identifying the proliferating cell population in irradiated tissues. For this reason, both antibodies were used to see whether the results of their use were complementary and/or interchangeable.

This study set out to evaluate retrospectively the proliferative activity of 31 cases of astrocytoma before and after radiotherapy. However, three cases were abandoned because they did not meet the criteria for inclusion in the study.

\section{Methods and materials}

SELECTION OF TISSUES

All of the patients had surgery for astrocytoma at the Regional Neurosurgical Unit, Brook Hospital, followed by radiotherapy, between 1978 and 1994, and were identified through departmental records. To select the appropriate blocks and confirm the diagnosis, haematoxylin and eosin stained slides of 
Table 1 Location of tumours

\begin{tabular}{lcc}
\hline Area & First surgery & Second surgery \\
\hline Frontal & 11 & 11 \\
Frontal-parietal & 1 & 1 \\
Parietal & 2 & 3 \\
Temporal & 7 & 7 \\
Occipital & 2 & 2 \\
Intraventricular & 1 & 1 \\
Cerebellum & 3 & 2 \\
Brainstem & 1 & 1 \\
Total & 28 & 28 \\
\hline
\end{tabular}

each case were reviewed by a consultant neuropathologist (RD). Sections that showed obvious astrocytic features and met the histological grading criteria of the World Health Organisation (WHO), ${ }^{17}$ and whose blocks had sufficient viable neoplastic tissue for immunohistochemistry, were chosen. For the purpose of this study, the specimens were classified as either low grade (WHO grades I and II) or high grade (WHO grades III and IV). The sections were also reviewed and regraded according to the Daumas-Duport, Scheithauer, O'Fallon, and Kelly method. ${ }^{18}$ Using the WHO classification, $73.2 \%$ were in the higher grade range (grades III and IV) and $26.8 \%$ were in the lower grade range (grades I and II). Similarly, using the Daumas-Duport system, the ranges were $69.6 \%$ and $30.4 \%$, respectively. There was good correlation between the two grading systems.

The group consisted of 18 men and 10 women, ranging in age from 22 to 65 years at initial surgery (mean, 41.3 years; median, 39.5 years) and 24 to 65 years at second surgery (mean, 43.4 years; median, 42.0 years). The 56 specimens were taken from sources located in the cerebral hemispheres and the cerebellum (table 1). One of the patients, who had a low grade initial tumour in the cerebellum, returned 18 months later with a high grade tumour in the parietal region. The second tumour had occurred in the path of the radiation field. The interval between initial and follow up surgery ranged from three months to six years and eight months.

The start of radiotherapy ranged from two to five weeks after surgery. Radiotherapy was given at six different centres, depending on the patient's area of residence. For most patients, the dosage ranged from 36 to $61 \mathrm{~Gy}$, and in most cases it was given in daily fractions over three to six weeks. Field sizes were designed to cover the tumour and a margin of approximately 2 to $3 \mathrm{~cm}$. One patient had a radical course of $60 \mathrm{~Gy}$ in 30 treatments and a stereotactic implant of three cranial catheters loaded with radioactive iodine seeds, which gave a further $50 \mathrm{~Gy}$ to the tumour. Another had 39.6 Gy to the whole brain, followed by a boost of $60 \mathrm{~Gy}$ to the tumour bed.

PREPARATION OF TISSUE SECTIONS FOR IMMUNOHISTOCHEMISTRY

The initial tissue samples came from primary surgery before radiotherapy and the second samples came from surgery after completion of radiotherapy. The fresh tissues were fixed in $10 \%$ neutral buffered formalin saline, followed by processing in paraffin wax. Sections of
3-4 $\mu \mathrm{m}$ were cut and floated on to poly-1-lysine coated slides (for PCNA) and 3-aminopropyltriethoxysilane (APES) (for MIB-1). The slides were then left to dry overnight at room temperature.

IMMUNOHISTOCHEMICAL TECHNIQUE

Sections were stained using a modified avidin-biotin technique. ${ }^{19}$ Briefly, sections were dewaxed, rehydrated and treated with $3 \%$ hydrogen peroxide in methanol to block endogenous peroxidase. Before incubation with the primary antibody, the MIB-1 specimens were pretreated in a microwave oven (750 watt Sanyo domestic oven used at its maximum power setting) immersed in $10 \mathrm{mM}$ citric acid buffer ( $\mathrm{pH}$ 6.0) for 12 minutes. Monoclonal NCL-PCNA PC10 (Novocastra Laboratories, Europath Ltd, Newcastle upon Tyne, UK) and MIB-1 (The Binding Site Ltd, Birmingham, UK) were both used at a dilution of $1 / 50$ and incubated for 30 minutes. The linker molecule, biotinylated rabbit antimouse IgG, was applied at a dilution of $1 / 400$ and the streptavidin-horseradish peroxidase complex (Dakopatts, Ely, UK) at a dilution of $1 / 200$, both for 30 minutes. All incubations were carried out in a darkened, humidified chamber at room temperature. To obtain maximum sensitivity, the primary and secondary antibodies were diluted in Tris buffered saline (TBS) containing bovine serum albumin. Peroxidase activity was revealed using 3,3'diaminobenzidine as the substrate, with a reaction time of five minutes. All washes were performed with TBS containing Triton-X 100. The preparations were lightly counterstained with haematoxylin, dehydrated, and mounted in Canada balsam.

IMMUNOHISTOCHEMICAL ASSESSMENT

The staining patterns were evaluated without knowledge of the histological grade and the clinical information. Initially, the whole section was scanned at low power to identify the area of highest positivity. This was followed by counting of the positive and negative nuclei in 10 fields at a magnification of $\times 100$ in oil immersion. To ensure reproducibility, a random selection of cases were recounted a few times. The numbers of cells varied according to the size of the specimen and the cell density of the tumour; they ranged from 400 to 1200 . The labelling indices were expressed as a percentage of the total cells counted and calculated as follows:

$$
\mathrm{LI}=100 \mathrm{p} / \mathrm{t}
$$

LI, labelling index; $p$, number of positive cells counted; $t$, total numbers of cells counted (positive and negative).

CONTROL MATERIAL

To ensure quantitative reproducibility, both positive and negative controls were included. Normal tonsil paraffin wax embedded material was used because it has an established level of reactivity with both PC10 and MIB-1. For negative controls, the primary antibody was replaced by TBS. 

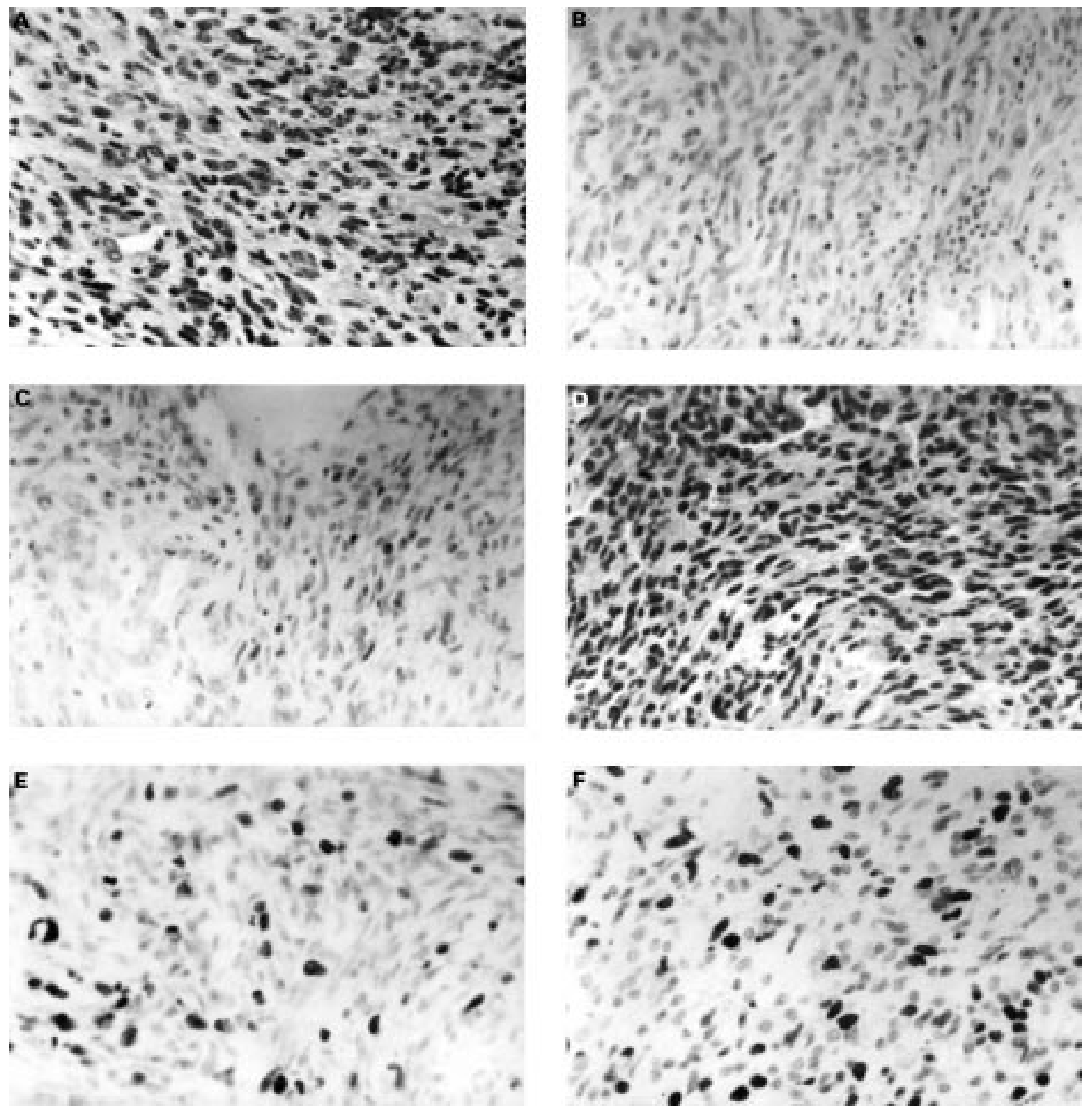

Figure 1 Anaplastic astrocytomas: $A-C$, pre-radiotherapy; $D-F$, post-radiotherapy. $A$ and $D$, haematoxylin and eosin stained; $B$ and $E, P C 10$ stained; $C$ and F, MIB-1 stained.

STATISTICAL ANALYSIS

Results are expressed as mean (standard error mean (SEM)). The relations between PC10 and MIB-1 were assessed using the Student's $t$ test and the correlation coefficient $(r)$. Microsoft Excel 5.0 was used for the analysis. p values of $<0.05$ were considered to be statistically significant.

\section{Results}

Both antibodies showed strong and discrete staining of the cell nuclei in the proliferative compartments of the astrocytomas. Although staining was found primarily in the nucleus, very rarely, faint cytoplasmic staining was also observed. Staining varied in intensity; however, all identifiable staining was recorded as positive. The pattern of staining for PC10 was dif- fuse, granular, or a mixture of both and for MIB-1, staining was mainly intense, diffuse, and coarsely granular, or had a clumped appearance (fig 1). On the whole, staining with MIB-1 had less variation of intensity and less "background reaction" than PC10. Therefore, in general, the staining intensity with MIB-1 was superior to that with PC10. Mitotic cells showed either diffuse staining or no staining at all. Normal brain cells adjacent to the tumour were not reactive. Tonsil immunoreactivity with PC10 and MIB-1 antibodies was consistently positive and was similiar to that of the astrocytoma sections. In the negative controls, no immunoreaction was observed.

The PCNA labelling indices ranged from 0 to $48.1 \%$ (mean, $18.5(2.7)$ ) at initial surgery and 0 to $75.1 \%$ (mean, $29.0(4.1)$ ) at second 


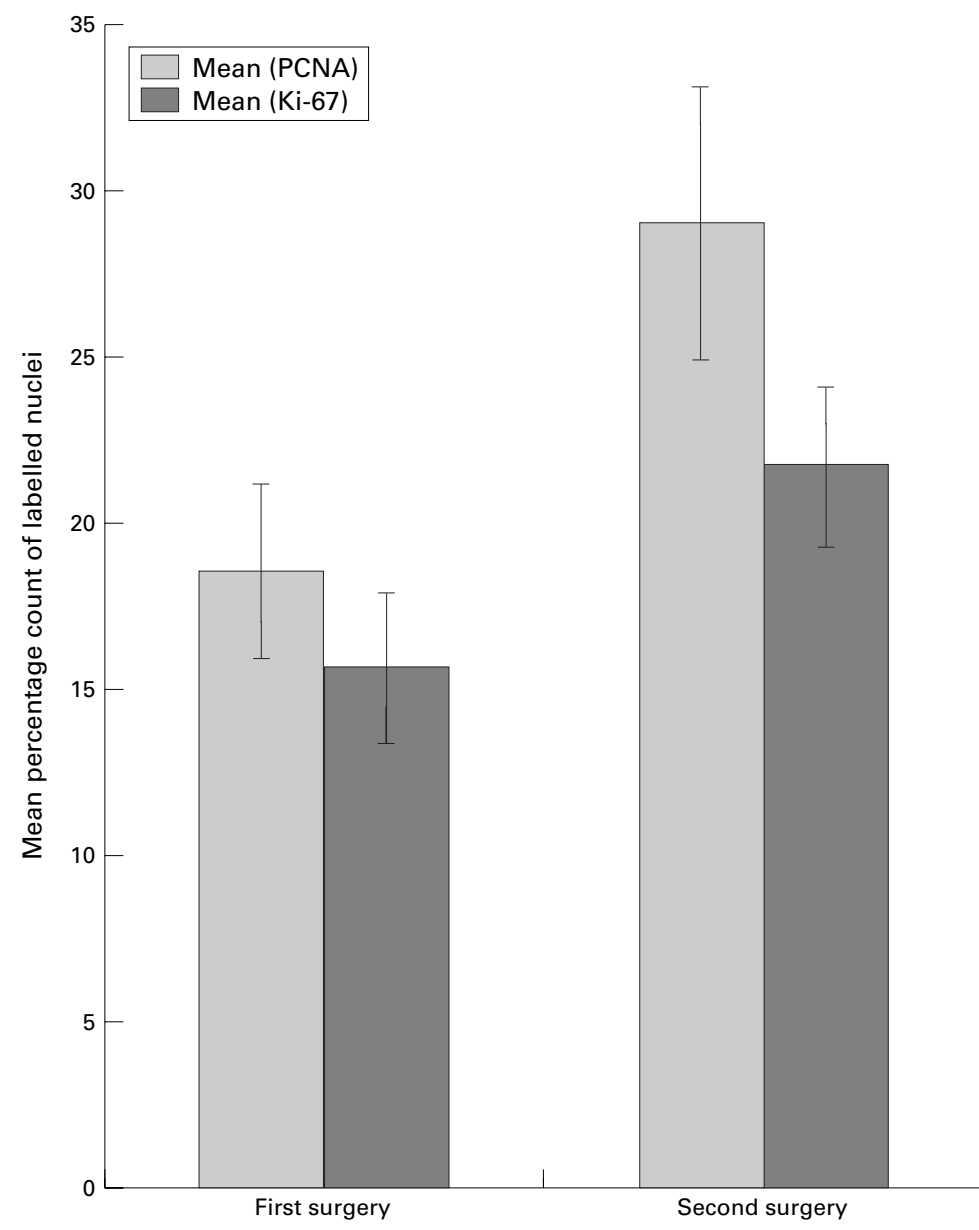

Figure 2 Mean proliferating cell nuclear antigen (PCNA) and Ki-67 labelling indices with standard errors in astrocytomas pre-radiotherapy and post-radiotherapy.

surgery. The Ki-67 labelling indices were 0 to $40 \%$ (mean, $15.6(2.2)$ ) and 0 to $56.6 \%$ (mean, 21.7 (2.43)), respectively. Mean PCNA counts were significantly higher than those with Ki-67 ( $<<0.01$ ) (fig 2). Only eight of the $28(28.6 \%)$ cases examined had a lower labelling index post-radiotherapy. There was good correlation between the initial and second surgery PCNA counts $(r=0.986)$. However, there was no correlation between the PCNA and MIB-1 counts.

\section{Discussion}

Both PC10 and MIB-1 appeared to identify proliferating cells in astrocytomas. Thus, the proliferative potential of astrocytoma cells can be measured in both current and archival conventionally processed tissues in any laboratory where immunohistochemistry is practised routinely and, therefore, it is of potential prognostic value. The higher labelling indices obtained with the antibody PC10 in this study were similar to those seen in hepatocellular carcinoma. ${ }^{20}$ This could be attributed to the long half life (20 hours) of PCNA and the expression of this antigen in proliferating, quiescent, and non-proliferating cells. ${ }^{2122}$ On the other hand, the antigen labelled by MIB-1 has a shorter half life and is catabolised rapidly after $M$ phase. ${ }^{14}$ Therefore, labelling with
PC10 might not accurately reflect the proliferative population of the cell cycle, because the PCNA epitope is expressed for a longer period of the cell cycle than $\mathrm{Ki}-67$. In addition, PC10 immunoreactivity is affected by heat and length of fixation time, ${ }^{1}$ while MIB- 1 is insensitive to these factors. ${ }^{23} \mathrm{~A}$ comparison of the staining intensity in an astrocytoma from the same patient showed MIB-1 staining to be more marked than PC10 (fig 1). On the basis of the above, and in our laboratory, MIB-1 appears to be superior to PC10 as an antibody for the detection of proliferation in astrocytomas.

Variations in the staining pattern and the labelling indices have been reported previously, ${ }^{1}$ and both might be attributable to technical factors, such as processing and fixation. We have also examined archival material with no standardisation of fixation time. Variation in staining was seen in different areas of the same specimen. This might be the result of focal differences in proliferative potential or lack of synchrony in the proliferation of neoplastic astrocytic cells. Indeed, similar results were found in trophoblastic disease. ${ }^{24}$ The method of establishing labelling indices used in this study, although time consuming, has been found to be reliable by many investigators. ${ }^{1525-27}$

Post-irradiated samples had greater labelling indices for both PCNA and Ki-67. In astrocytomas, the degree of sensitivity to radiation depends on histological grading and the more malignant the astrocytoma, the greater is the intrinsic radioresistance. ${ }^{28-30}$ Most of the post-irradiated specimens in this series were in the higher grade range (25 of $30)$. There is a more favourable clinical outcome for patients who have had postoperative radiotherapy, compared with those who have not. ${ }^{28}{ }^{31-33}$ For this reason, radiotherapy is the most widely used treatment. However, the use of radiotherapy remains controversial and there are arguments for and against its use in the management of astrocytoma. The dose has to be limited, because normal brain tissue cannot safely tolerate more than $60 \mathrm{~Gy}^{34}$ Also, radiobiological studies have shown that, in general, astrocytoma cells proliferate slowly and are not very sensitive to radiation. Thus, the single daily fraction of radiation, which is targeted at a slow proliferating pool of neoplastic cells, will only damage a small number of those cells. ${ }^{35}$ In this respect, mitotic cells are the most likely ones to be damaged during the administration of radiotherapy. Because most of the patients in this study were treated by daily fractions, this could account for the higher labelling indices in the post-radiation specimens. Delivery of radiation by other means to overcome these limitations has been developed, but all methods have certain advantages and disadvantages. ${ }^{36}$ Repair, reoxygenation, reassortment in the cell cycle, and repopulation are the biological processess that take place during a course of fractionated radiotherapy. ${ }^{37}$ The first three processes favour radiotherapy and are beneficial in decreasing tumour 
growth; the latter process has the opposite effect. ${ }^{38}$ The extent of repopulation depends not only on tumour growth kinetics but also on the length, frequency, and number of interruptions during therapy. The subjects in this study received radiotherapy at a centre nearest to their homes. Thus, six radiotherapy centres were involved and it is not known to what extent therapy was maximised. "Conventional fractionation" is known to vary depending on the radiotherapist and the centre. $^{32} 33$

Radiation could be responsible for the more aggressive nature of astrocytomas. Thus, the link between radiation and the increased proliferation needs to be explored further. Accelerated tumour cell proliferation rates have been reported, and the results of a number of clinical trials on tumour cell kinetics are awaited to ascertain whether accelerated or conventional radiotherapy regimens are the cause. $^{39}$

Choucair et al reported that more than $90 \%$ of patients with malignant glioma had recurrence of their tumour at the original site after radiotherapy. ${ }^{40}$ All of the astrocytomas we examined were pre-irradiated and postirradiated specimens. Therefore, it could be argued that radiation therapy was responsible not only for the higher proliferation index in the recurrent specimens but also for the recurrence.

It cannot be assumed that any tumour is static in its cytology. Furthermore, the progress of advancing degradation in astrocytomas is well documented. ${ }^{41}{ }^{42}$ Recent studies have shown that astrocytomas evolve and progress as a result of genetic alterations. ${ }^{43-46}$ It may also be important to take the size of the biopsy taken from these lesions into account and to establish whether there is a correlation between patient survival, response to therapy, and PCNA and Ki-67 labelling indices. This study is being extended to include these factors.

1 Hall PA, Levison DA, Woods AL, et al. Proliferating cell nuclear antigen (PCNA) immunolocalisation in paraffin sections: an index of cell proliferation with evidence of deregulated expression in some neoplasms. I Pathol 1990;162:285-94

2 Gerdes J, Schwab LL, Leuke H, et al. Production of a mouse monoclonal antibody reactive with a human nuclear antigen associated with cell proliferation. Int $\mathcal{f}$ Cancer 1983;31:13-20.

3 Robbins B, de la Vega D, Ogata K, et al. Immunohistochemical detection of proliferating cell nuclear antigen in solid human malignancies. Arch Pathol Lab Med 1987;111: 841-5.

4 Gerdes J. Ki-67 and other proliferation markers useful for immunohistological diagnostic and prognostic evaluations in human malignancies. Semin Cancer Biol 1990;1:199 206.

5 Jain S, Filipe MI, Hall PA, et al. Prognostic value of proliferating cell nuclear antigen in gastric carcinoma. $\mathcal{F}$ Clin Patho 1991;44:655-9.

6 Woods AL, Hall PA, Shepherd NA, et al. The assessment of proliferating cell nuclear antigen (PCNA) immunostaining in primary gastrointestinal lymphomas and its relationship to histological grade, $S+G_{2}+M$ phase fraction (flow cytometric analysis) and prognosis. Histopathology 1991;18: $21-7$

7 Kamel D, Turpeenniemi-Hujanen T, Vähäkangas K, et al. Proliferating cell nuclear antigen but not p 53 or human Proliferating cell nuclear antigen but not p 53 or human
papillomavirus DNA correlates with advanced clinical papillomavirus DNA correlates with advanced clinical
stage in renal cell carcinoma. Histopathology 1994;25:339stage
8 Waseem NH, Lane DP. Monoclonal antibody analysis of the proliferating cell nuclear antigen (PCNA). Structural conservation and the detection of a nucleolar form. $7 \mathrm{Cell} \mathrm{Sci}$ 1990;96:121-9.

9 Cattoretti G, Becker MGH, Key G, et al. Monoclonal antibodies against recombinant parts of the $\mathrm{Ki}-67$ antigen (MIB-1 and MIB-3) detect proliferating cells in microwave processed formalin-fixed paraffin sections. F Pathol 1992; 168:357-63

10 Linden MD, Torres FX, Kubus J, et al. Clinical application of morphologic and immunocytochemical assessments of cell proliferation. Am f Clin Pathol 1992;97(suppl 1):S4-S13.

11 Bravo R, MacDonald-Bravo H. Changes in the nuclear distribution of cyclin (PCNA) but not its synthesis depend on tribution of cyclin (PCNA) but not its synth

12 Bravo R, MacDonald-Bravo H. Existence of two populations of cyclin/proliferating cell nuclear antigen during the cell cycle: association with DNA replication sites. $f$ Cell Biol $1987 ; 105: 1549-54$.

13 Gerdes J, Lemke H, Baisch H, et al. Cell cycle analysis of a cell proliferation-associated human nuclear antigen defined by monoclonal antibody Ki-67. F Immunol 1984;133:1710-5.

14 van Dierendonck JH, Keijzer R, van de Velde $\mathrm{CJH}$, et al. Nuclear distribution of the Ki-67 antigen during the cell cycle: comparison with growth fraction in human breast cancer cells. Cancer Res 1989;49:2999-3006.

15 Siddiqi SN, Provias J, Laperriere N, et al. Effects of iodine-

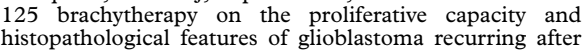
initial therapy. Neurosurgery 1997;40:910-18.

16 Valente G, Orecchia R, Gandolfo S, et al. Can Ki-67 immunostaining predict response to radiotherapy in ora

17 Kleihues P, Burger PC, Scheithauer BW. W.H.O. histological typing of tumours of the nervous system. 2nd edn. Berlin: Springer-Verlag, 1993.

18 Daumas-Duport C, Scheithauer BW, O'Fallon J, et al. Grading of astrocytomas: a simple and reproducible method. Cancer 1988;62:2152-65.

19 Hsu S-M, Raine L, Fanger H. Use of avidin-biotinperoxidase complex (ABC) in immunoperoxidase techniques: a comparison between $\mathrm{ABC}$ and unlabelled antibody (PAP) procedures. F Histochem Cytochem 1981;29: 577-80.

$20 \mathrm{Ng}$ IOL, Na J, Lai ECS, et al. Ki-67 antigen expression in hepatocellular carcinoma using monoclonal antibody MIB-1. A comparison with proliferating cell nuclear antigen. Am f Clin Pathol 1995;104:313-18

21 Diebold J, Dopfer K, Lai M, et al. Comparison of different monoclonal antibodies for the immunohistochemical assessment of cell proliferation in routine colorectal biopsy specimens. Scand $\mathcal{F}$ Gastroenterol 1994;29:47-53.

22 Lynch DAF, Clarke AMT, Jackson P, et al. Comparison of labelling by bromodeoxyuridine, MIB-1, and proliferating cell nuclear antigen in gastric mucosal biopsy specimens. $\mathcal{F}$ Clin Pathol 1994;47:122-5.

23 Schwarting R. Little missed markers and Ki-67. Lab Invest 1993;68:597-9.

24 Cheung ANY, Ngan HYS, Chen WZ, et al. The significance of proliferating cell nuclear antigen in human trophoblastic disease: an immunohistochemical study. Histopathology 1993;22:565-8

25 Revesz T, Alasanjari N, Darling, et al. Proliferating cell nuclear antigen (PCNA): expression in samples of human astrocytic gliomas. Neuropathol Appl Neurobiol 1993;19: 152-8.

26 Carr NJ, Monihan JM, Nzeako UC, et al. Expression of proliferating cell nuclear antigen in hyperplastic polyps, adenomas and inflammatory cloacogenic polyps of the large intestine. f Clin Pathol 1995;48:46-52.

27 McCormick D, Yu C, Hobbs C, et al. The relevance of antibody concentration to the immunohistochemical quantification of cell proliferation-associated antigens. Histopatholcation of cell prolife $1993 ; 22: 543-7$.

28 Taghian A, Suit H, Pardo F, et al. In vitro intrinsic radiation sensitivity of glioblastoma multiforme. Int $\mathcal{F}$ Radiat Oncol Biol Phys 1993;23:55-62.

29 Taghian A, Ramsay J, Allunis-Turner J, et al. Intrinsic radiation sensitivity may not be the major determinant of the poor clinical outcome of glioblastoma multiforme. Int $\mathcal{F}$ Radiat Oncol Biol Phys 1993;25:243-9.

30 Leibel SA, Scott CB, Loeffler JC. Contemporary approaches to the treatment of malignant gliomas in radiation therapy. Semin Oncol 1994;21:198-219.

31 Walker MD, Green SB, Byar DP, et al. Randomised comparisons of radiotherapy and nitrosoureas for the treatment of malignant gliomas after surgery. $N$ Engl $7 \mathrm{Med}$ 1980;303:1323-9.

32 Kristiansen K, Hagen S, Kolleveld $\mathrm{T}$, et al. Combined modality therapy of operated astrocytomas grade III and V. Confirmation of the value of of post-operative irradiation and lack of potentiation of bleomycin on survival time: a prospective multicentral trial of the Scandinavian Glioblastoma Group. Cancer 1981;47:649-52.

33 Leibel SA, Sheline GE. Radiotherapy in the treatment of cerebral astrocytomas. In: Thomas DGT, ed. Neurooncology, primary malignant brain tumours. London: Edward Arnold, 1990:193-221.

34 Yoshii Y, Takano S, Tsurushima $\mathrm{H}$, et al. Normal brain damage after radiotherapy of brain tumours. Clin Oncol 1991;3:278-82.

35 Kornblith PL. Control of tumour cell proliferation and radiation therapy. Surg Neurol 1995;43:393-4. 
36 Maughan TS. Basic principles of radiotherapy for surgical oncologists 4 - fractions, photons and the future. Eur $f$
Surg Oncol 1994;20:484-92.

37 Hall EJ. Radiobiology for the radiologist. 4th edn. Philadelphia: JB Lippincott, 1944.

38 Withers HR, Taylor JMG, Maciejewski B. The hazard of accelerated tumour clonogen repopulation during radiotherapy. Acta Oncol 1988;27:132.

39 Lawton PA. Predicting the response to radiotherapy. Clin Oncol 1993;5:193-4.

40 Choucair AK, Levin VA, Gutin PH, et al. Development of multiple lesions during radiation therapy and chemotherapy in patients with gliomas. 7 Neurosurg 1986;65:654-8.

41 Salazar OM, Rubin P. The spread of glioblastoma volume. Int f Radiat Oncol Biol Phys 1976;1:627-37.
42 Russell DS, Rubinstein LJ. Tumours of central neuroepithelial origin. In: Pathology of tumours of the nervous system. London: Edward Arnold, 1989:95-161.

43 Henson JW, Schnitker BL, Correa KM, et al. The retinoblastoma gene is involved in malignant progression of astrocytomas. Ann Neurol 1994;36:714-21.

44 Ohgaki H, Schäuble B, zur Hausen A, et al. Genetic alterations associated with the evolution and progression of astrocytic brain tumours. Virchows Arch 1995;427:113-18.

45 Watanabe K, Tachibana O, Sato K, et al. Overexpression of the EGF receptor and p53 mutations are mutually exclusive in the evolution of primary and secondary glioblastomas. Brain Pathol 1996;6:217-24.

46 Louis DN. A molecular genetic model of astrocytoma histopathology. Brain Pathol 1997;7:755-64. 